\title{
Memory Enhancement from Two Weeks' Exposure to North American Ginseng Extract HT1001 in Young and Middle Aged Healthy Adults
}

\author{
Sharla K. Sutherland ${ }^{1, *}$, Scot E. Purdon ${ }^{2}$, Chien-Tsai Lai ${ }^{1,2}$, Li-Juan Wang ${ }^{3}$, Gan-Zhong Liu ${ }^{4}$ and \\ Jacqueline J. Shan ${ }^{1}$ \\ ${ }^{I}$ Afexa Life Sciences, Edmonton, Alberta, Canada \\ ${ }^{2}$ Department of Psychiatry, University of Alberta, Edmonton, Alberta, Canada \\ ${ }^{3}$ Department of Pharmacology, Capital Medical University, Beijing, China \\ ${ }^{4}$ Institute of Clinical Medical Sciences, China-Japan Friendship Hospital, Beijing, China
}

\begin{abstract}
In vivo, in vitro, and animal learning studies have reported neuroactive effects of Rb1 and Rg1 ginsenosides that may be relevant to human learning and memory. The objective of this study was to assess potential learning and memory benefits of HT1001, a standardized proprietary North American ginseng (Panax quinquefolius) extract containing $\mathrm{Rb} 1, \mathrm{Rg} 1$ and other important ginsenosides, in healthy volunteers. Neuropsychological assessments were conducted using the Clinical Memory Scale (CMS), which has two parallel forms for baseline and post-treatment assessments. A young adult sample (YAS, $n=10$ ) and a middle aged sample (MAS, $n=10$ ) completed the CMS at baseline and again after 14 days' exposure to 200mg HT1001 daily. The CMS Memory Quotient (MQ) showed significant main effects of time, with higher CMS-MQ on the second assessment compared to the first, and of age group, with the YAS performing better than the MAS. There was no interaction between time and age group. Secondary analyses indicated benefits for both groups on free recall of word lists, cued recall of word pairs, and recognition of figures, and benefits in the YAS but not the MAS on free recall of pictures. Taken together, the results suggest that memory, as measured with the CMS-MQ, was significantly improved with open-label HT1001. While practise effects cannot be completely ruled out, the results presented here are exciting and timely given our increasingly ageing population, and provide preliminary support for a prospective placebo-controlled examination of HT1001 on learning and memory.
\end{abstract}

Keywords: North American ginseng extract, HT1001, Clinical Memory Scale, memory, learning.

\section{INTRODUCTION}

A staple of traditional Chinese medicine for thousands of years, the ginseng root has a wide spectrum of presumed health-related benefits recently subjected to contemporary scientific methods of validation [1,2]. The longstanding presumption that ginseng may improve cognitive skills has become particularly relevant as a result of in vitro and in vivo demonstrations of neural growth enhancement and inhibition of neural degeneration [3-5]. Technological improvements in the delineation and quantification of the neuroactive components of the ginseng root have facilitated this investigation by identifying several ginsenosides apparently relevant to the consolidation of new memories, including Rb1, which has been shown to modulate acetylcholine release and re-uptake in rat brain [6] and to induce mRNA expression of choline acetyltransferase and nerve growth factor in rat brain [7], and $\mathrm{Rg} 1$, which has been reported to induce proliferation and differentiation of neural progenitor cells in the dentate gyrus of the hippocampus of adult mice [8]. Animal behaviour

*Address correspondence to this author at the Afexa Life Sciences, 9604$20^{\text {th }}$ Avenue NW, Edmonton, AB Canada T6N 1G1, Canada; Tel: +780432-0022; Fax: +780-432-7772; E-mail: SSutherland@afexa.com studies have also implicated $\mathrm{Rb} 1$ and $\mathrm{Rg} 1$ in memory enhancement [9] and in the prevention of acquired memory deficits [10-13].

The preclinical data were sufficient to stimulate several clinical investigations of learning, but the results have been inconclusive regarding the beneficial effects of sustained exposure to ginseng extracts in humans. Two double-blind placebo-controlled investigations have reported memory enhancement with ginseng $[14,15]$ but a third did not [16]. Also, an assessment of memory among self-proclaimed ginseng users reported no benefit relative to a control sample [17]. The ambiguous results may relate to differences in samples, ginseng extracts, or measurement strategy. For example, beneficial effects were observed in older infirm samples $[14,15]$ and in individuals with Alzheimer's disease [18], but not in younger healthier samples [16,17]. Benefits were observed with relatively high daily doses of extract [14], but not lower doses [16]. Also, benefits were observed on traditional standardized tests of new verbal learning [14] and on a small standardized battery of tests weighted toward new verbal learning [15], but gains were not observed on experimental measures [17], or in the savings scores of standardized tests of delayed figure or text recall [16]. 
Although the variability of methods across studies precludes a confident inference regarding memory enhancement with ginseng extracts, these experiments suggest potential value of an examination of a precise formulation of $\mathrm{Rb} 1$ and Rg1 ginsenosides using standardized tests of verbal and nonverbal memory with parallel groups of younger and older healthy control subjects. Indeed, the importance of standardizing natural product extracts has been highlighted in works by Dr. Basu and others in recent years [19-21]. HT1001 (REMEMBER-FX®; Afexa Life Sciences Inc., Edmonton, Alberta, Canada) is a proprietary North American ginseng extract developed using patented chemical and biological standardization technology (ChemBioPrint ${ }^{\circledR}$; Afexa Life Sciences Inc.) containing known levels of active ginsenosides (Rb1, Rb2, Rc, Rd, Re and Rg1) totalling 13-20\%. $100 \mathrm{mg}$ HT1001 provides the equivalent of $500 \mathrm{mg}$ of North American ginseng dried root, and one capsule of HT1001 consumed twice daily is consistent with the therapeutic doses described in Traditional Chinese Medication references $[22,23]$. In the preliminary prospective open-label examination described here, performance on the Clinical Memory Scale (CMS) [24] was examined before and after 2 weeks' open-label exposure to HT1001(100 mg twice daily) in a young adult and a middle-aged adult sample of healthy volunteers. The results suggest that a larger-scale placebocontrolled study investigating the memory-enhancing effects of HT1001 is certainly warranted.

\section{MATERIALS AND METHODOLOGY}

\section{Subjects}

The current study was in strict compliance with the regulations of the Capital Medical University in Beijing, which are entirely consistent with the Helsinki Declaration. All participants provided informed consent to participate in this investigation. Ten younger and ten older right-handed participants were recruited primarily from medical students and mixed staff, respectively, at the Capital Medical University in Beijing. The young-adult sample (YAS) was 20 to 24 years of age $($ mean $=21.30, S D=1.25)$, and the middle-age sample (MAS) was 45 to 65 years of age (mean $=55.20, S D$ $=5.73)$. Years of education were similar in the YAS (mean $=$ $14.20, S D=0.63)$ and MAS (mean $=13.40, S D=1.26)$, but the proportion of men tended to be higher in the YAS $(50 \%)$ compared to the MAS $(20 \%), \chi^{2}(1)=1.98, p=0.160$. A routine examination including laboratory tests was conducted at the beginning of the study to ensure that all participants were in good health.

\section{The Clinical Memory Scale (CMS)}

The CMS was completed at baseline and again after consumption of one $100 \mathrm{mg}$ capsule of HT1001 in the morning and a second in the evening, each day for 14 consecutive days. The CMS was developed in Mandarin by the Chinese Academy of Sciences and standardized on a sample of 3310 normal controls between the ages of 20 and 89 years [24]. The CMS consists of five tests of memory including free recall of word lists with distracters, cued recall of word pairs, free recall of pictures, recognition of meaningless figures, and cued recall of facts associated with unfamiliar portraits. Two alternate forms are available with good psychometric properties and inter-test reliability $(r=0.85)[24,25]$. The present application followed the test manual recommendation of Form A at baseline and Form B at follow up. The CMS has been applied with success in studies of cerebrovascular disease [26], multiple sclerosis [27], mild cognitive impairment [28], dementia [29], and genetic polymorphisms associated with Alzheimer's disease [28]. The CMS was also applied with success in at least one prospective examination of treatment effects, demonstrating no improvement through time (i.e. no practise effect) on the alternate forms of the CMS in the absence of treatment, but significant memoryenhancement after 10 days of sustained cognitive training [30].

\section{Statistical Analysis}

The primary hypothesis that HT1001 would enhance memory was examined by subjecting the CMS Memory Quotient (MQ) to repeated measures analysis of variance with time as a within subject variable and age group as a between subject variable. A secondary analysis to depict subtest characteristics of the anticipated change was undertaken by subjecting the five scaled subtest scores to a multivariate repeated measures analysis of variance with time as a within subject variable, and age group as between subject variable. All tests were two-tailed with an alpha of 0.05 except under Bonferroni correction as described below.

\section{RESULTS}

A substantial improvement in CMS-MQ scores was observed between baseline $($ mean $=110.60, S D=11.78)$ and follow up $($ mean $=122.73, S D=14.57), F(1,18)=30.56, p<$ 0.001 . The YAS demonstrated higher average (i.e. combined pre- and post-treatment) CMS-MQ scores (mean $=125.40$, $S D=9.08)$ than the MAS (mean $=107.93, S D=13.61)$, $F(1,18)=20.17, p<0.001$ (see Fig. 1). There was no interaction between time and age group, with similar improvement in CMS-MQ score in the YAS (mean improvement = $13.70, S D=5.30$ ) and MAS (mean improvement $=10.55$, $S D=12.82$ ). Relative to the standardization sample [24], the YAS CMS-MQ scores were above average to high prior to HT1001 treatment and well above average post-treatment, whereas the MAS CMS-MQ scores were average prior to HT1001 treatment and high average after sustained HT1001 exposure.

The secondary analysis also documented a main effect of age group, $F(5,14)=15.11, p=0.00003$, and time, $F(5,14)=$ $10.52, p=0.0002$, as well as an interaction between age group and time, $F(5,14)=0.659, p=0.002$. The univariate analyses showed a main effect of age group on all variables, with the YAS outperforming the MAS on all five subtests (see Table 1). Main effects of time were observed on free recall of word lists, cued recall of word pairs, and recognition of figures (all $p$ 's $<0.0006$ and significant after Bonferroni correction), but not free recall of pictures or cued recall of facts about portraits. There were no interactions between age group and time for cued recall of word pairs or recognition of meaningless figures; both the YAS and the MAS showed significant improvements between baseline and follow up. There were interactions between age group and time for free recall of word lists and free recall of pictures, and a trend towards an interaction on cued recall of facts about portraits. In contrast, free recall of pictures showed a gain 


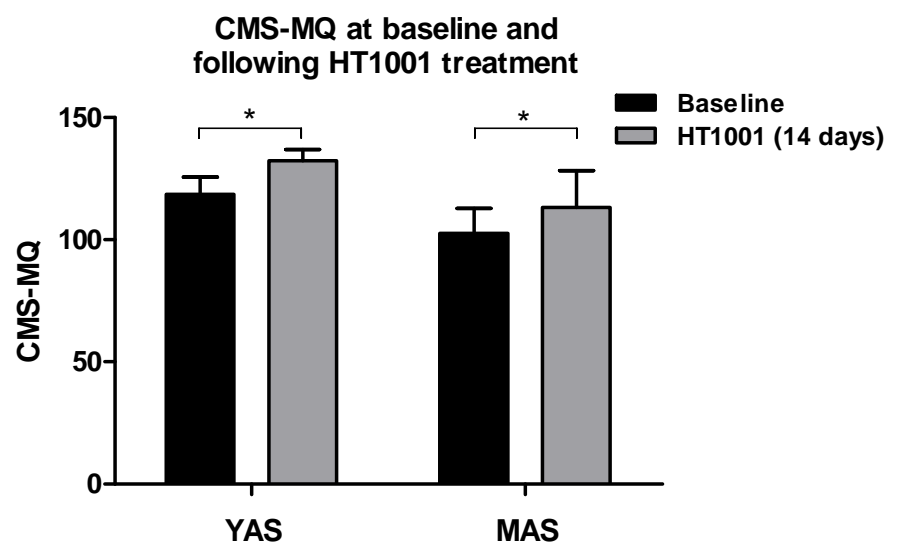

* denotes a significant difference between groups $(\mathrm{p}<0.05)$.

Fig. (1). Clinical Memory Scale Memory Quotients (CMS MQ) for the Young Adult Sample (YAS) and the Middle Aged Sample (MAS) before (black bars) and after 14 days' of HT1001 (gray bars) (mean \pm SD).

Table 1. Scaled Scores and Memory Quotients (mean \pm SD) for the Young Adult Sample (YAS) and the Middle Aged Sample (MAS) at Baseline and after 14 days' Exposure to HT1001

\begin{tabular}{|l|c|c|c|c|c|c|}
\hline \multirow{2}{*}{} & \multicolumn{2}{|c|}{ Young Adult Sample (YAS) $n=10$} & \multicolumn{2}{c|}{ Middle Aged Sample (MAS) $n=10$} \\
\cline { 2 - 6 } & Baseline & 2 Weeks & Difference & Baseline & 2 Weeks & Difference \\
\hline \hline Free Recall of Word Lists & $26.30 \pm 3.06$ & $30.40 \pm 2.27$ & $4.10 \pm 3.14^{\mathrm{a}, \mathrm{b}}$ & $20.20 \pm 4.05$ & $26.55 \pm 4.41$ & $6.35 \pm 5.00^{\mathrm{a}, \mathrm{b}}$ \\
\hline Cued Recall of Word Pairs & $30.40 \pm 3.75$ & $34.55 \pm 3.32$ & $4.15 \pm 3.79^{\mathrm{a}}$ & $17.90 \pm 3.84$ & $23.05 \pm 5.25$ & $5.15 \pm 3.56^{\mathrm{a}}$ \\
\hline Free recall of Pictures & $27.40 \pm 4.50$ & $31.20 \pm 2.10$ & $3.80 \pm 5.09^{\mathrm{a}, \mathrm{b}}$ & $21.80 \pm 5.16$ & $20.50 \pm 4.33$ & $-1.30 \pm 5.01^{\mathrm{b}}$ \\
\hline Recognition of Figures & $27.30 \pm 3.97$ & $30.45 \pm 2.49$ & $3.15 \pm 3.31^{\mathrm{a}}$ & $18.80 \pm 5.37$ & $21.95 \pm 4.91$ & $3.15 \pm 3.70^{\mathrm{a}}$ \\
\hline Cued Recall of Portrait Facts & $26.30 \pm 3.22$ & $28.00 \pm 3.65$ & $1.70 \pm 2.90$ & $18.20 \pm 4.42$ & $15.50 \pm 6.49$ & $-2.70 \pm 6.25$ \\
\hline Memory Quotient & $118.55 \pm 7.02$ & $132.25 \pm 4.53$ & $13.70 \pm 5.30^{\mathrm{a}}$ & $102.65 \pm 10.17$ & $113.20 \pm 15.02$ & $10.55 \pm 12.82^{\mathrm{a}}$ \\
\hline
\end{tabular}

${ }^{a}$ within group difference between baseline and follow up, $p<0.05$.

${ }^{\mathrm{b}}$ group $\mathrm{x}$ time interaction, $p<0.05$.

only in the YAS, $t(9)=2.36, p=0.043$, significantly different from the trend towards a reduction apparent in the MAS, $t(18)=2.26, p=0.037$. The trend interaction observed with cued recall of facts about portraits resulted from a trend towards improvement in the YAS, $t(9)=1.86, p=0.097$, that was almost significant relative to the slight reduction observed in the MAS, $t(18)=2.02, p=0.059$.

\section{DISCUSSION}

Previous examinations of potential memory enhancing effects from ginseng suggest that sustained high doses of extracts administered to infirm elderly samples and to individuals with Alzheimer's disease may produce benefits on standardized tests of new learning and memory [14, 15, 18]. The current results suggest that the benefits may also be achieved with 14 days' exposure to a standardized ginsenoside formulation in healthy young and middle aged adults. Despite high baseline scores, particularly in the young adult sample, significant improvements were observed in the CMS-MQ of both samples. The most robust gains were observed on free recall of word lists, cued recall of word pairs, and recognition memory of meaningless figures. The young adult sample also showed a significant improvement in free recall of pictures and a trend towards gains in cued recall of facts about portraits, though the middle aged sample did not show similar improvements. The more robust gains observed in the young adult sample run contrary to speculation from earlier results that the magnitude of change might directly relate to the severity of infirmity at baseline. Subsequent investigations will be required to address the lack of any gain observed in the middle aged sample on free recall of pictures or free recall of facts about portraits, both of which appear to have additional demands for elaborative processing from visual stimuli to verbal encoding not required in the other three subtests.

The current results suggest that the discrepancies apparent in prior studies are not likely related to degree of baseline infirmity, but rather to characteristics of the examined ginseng extract or to the methods applied for the quantification of changes in learning and memory through time. The extracts in the prior studies were not precisely characterized in relation to potentially relevant ginsenosides, but improvement in word list learning was reported after exposure to $1500 \mathrm{mg}$ per day of Korean Ginseng extract [14], a Panax 
ginseng that would contain $\mathrm{Rb} 1$ and $\mathrm{Rg} 1$, but in unknown quantities. The positive results obtained in the present study may thus suggest that HT1001, a standardized extract containing measured quantities of Rb1 and Rg1 and other ginsenosides, possesses the necessary amounts of neuroactive ginsenosides to elicit memory enhancement from a ginseng extract whereas other ginseng products may not. Furthermore, with the exception of one report of beneficial effects of ginseng on the Randt Memory Test [15], the cognitive instruments applied in prior studies were not well characterized [14], appear to be study-specific [17], or produced atypical results that did not permit conventional reporting [16]. The positive results obtained in the present study underscore the value of standardized instrumentation in the demonstration of beneficial effects of ginseng extracts. Moreover, the Randt Memory Test, previously applied with success [15], is heavily weighted on verbal learning, and the most notable benefits observed in the present study were obtained on two verbal tasks. The robust improvement observed on a test of recognition of meaningless figures would caution against overly specific inferences about the cognitive domains sensitive to ginseng effects. However, it would be wise in subsequent investigations to maximize the likelihood of positive results by investigation of potential improvements in verbal learning and memory from well standardized extracts.

Although substantial benefits of ginseng on learning and memory were observed in the present study, there were several limitations that should be addressed in future studies. Importantly, a placebo control group was not included in the current study, and should be incorporated into future work to ensure that the improvements in memory function were not the result of a practice effect. Alternative forms of the CMS were used at baseline and follow up in the present study, which would undoubtedly attenuate or eliminate any beneficial effects of prior exposure to the test materials, however an appeal to the CMS manual was unhelpful in this regard; while a slight $2 \mathrm{MQ}$ disadvantage on Form B relative to Form A was reported in a counterbalanced design, the authors did not publish the absolute scores from their testretest groups [24]. A prospective study of heroin users reported no improvement from Form A to Form B in the absence of 10 days of cognitive training [31], but generalization to healthy control subjects remains tentative. The current preliminary investigation also studied a small number of participants, and a larger sample size would increase the power of the analysis. In addition, although the improvement in verbal list learning, verbal paired associate learning, and figure learning observed here suggests that the benefits may be robust across different age groups with average to above average baseline scores, further investigation will be helpful to generalize the benefits to picture recall and memory for facts about portraits beyond the relatively high functioning young adult medical students.

While the present study is the first to demonstrate a beneficial effect of sustained exposure of Panax Ginseng on memory in healthy adults, acute administration of ginseng extracts has been shown to improve a number of memory and cognitive domains in younger healthy adults, including improvements in working memory and increases in the speed of memory performance and the accuracy of attentional tasks [32-35]. As is observed with longer-term administration, however, results appear to be conflicting, likely resulting from experimental factors (such as the dose of ginseng consumed and the amount of time between ginseng consumption and cognitive testing). Further studies are certainly warranted to investigate the acute effects of a single dose of HT1001 on memory and cognition in healthy adults.

\section{CONCLUSIONS}

Ginseng has been used in traditional Chinese medicine for thousands of years, and it is gaining popularity in Western countries for a variety of health-related benefits. Human studies are few in number and their results have been inconclusive, but the benefits to learning and memory of two weeks' exposure to HT1001 observed in the present study clearly support the value of a larger-sample prospective assessment of HT1001 effects on standardized tests of learning and memory that includes a parallel placebo control arm, preferably within a double-blind cross-over design.

\section{ACKNOWLEDGEMENTS}

Afexa Life Sciences Inc, Edmonton, Alberta, Canada, provided financial support to Dr. Liu and Ms. Wang for data collection in this study. Drs. Sutherland, Lai and Shan are employees of Afexa. Dr. Purdon currently holds a research grant from Afexa. The authors have no other financial relationships relating to this work.

\section{REFERENCES}

[1] Radad K, Gille G, Liu L, Rausch WD. Use of ginseng in medicine with emphasis on neurodegenerative disorders. J Pharmacol Sci 2006; 100: 175-86.

[2] Cheng Y, Shen LH, Zhang JT. Anti-amnestic and anti-aging effects of ginsenoside $\mathrm{Rg} 1$ and $\mathrm{Rb} 1$ and its mechanism of action. Acta Pharmacol Sin 2005; 26: 143-9.

[3] Himi T, Saito H, Nishiyama N. Effect of ginseng saponins on the survival of cerebral cortex neurons in cell cultures. Chem Pharm Bull (Tokyo) 1989; 37: 481-4.

[4] Kim YC, Kim SR, Markelonis GJ, Oh TH. Ginsenosides Rb1 and $\mathrm{Rg} 3$ protect cultured rat cortical cells from glutamate-induced neurodegeneration. J Neurosci Res 1998; 53: 426-32.

[5] Lim JH, Wen TC, Matsuda S, et al. Protection of ischemic hippocampal neurons by ginsenoside Rb1, a main ingredient of ginseng root. Neurosci Res 1997; 28: 191-200.

[6] Benishin CG. Actions of ginsenoside Rb1 on choline uptake in central cholinergic nerve endings. Neurochem Int 1992; 21: 1-5.

[7] Salim KN, McEwen BS, Chao HM. Ginsenoside Rb1 regulates ChAT, NGF and trkA mRNA expression in the rat brain. Brain Res Mol Brain Res 1997; 47: 177-82.

[8] Shen LH, Zhang JT. Ginsenoside Rg1 promotes proliferation of hippocampal progenitor cells. Neurol Res 2004; 26: $422-8$.

[9] Mook-Jung I, Hong HS, Boo JH, et al. Ginsenoside Rb1 and Rg1 improve spatial learning and increase hippocampal synaptophysin level in mice. J Neurosci Res 2001; 63: 509-15.

[10] Zhang JT, Qu ZW, Liu Y, Deng HL. Preliminary study on antiamnestic mechanism of ginsenoside Rg1 and Rb1. Chin Med J (Engl) 1990; 103: 932-8.

[11] Benishin CG, Lee R, Wang LC, Liu HJ. Effects of ginsenoside Rb1 on central cholinergic metabolism. Pharmacology 1991; 42: 223-9.

[12] Wang XY, Chen J, Zhang JT. Effect of ginsenoside Rg1 on learning and memory impairment induced by beta-amyloid peptide(2535 ) and its mechanism of action. Yao Xue Xue Bao 2001; 36: 1-4.

[13] Sloley BD, Pang PK, Huang BH, et al. American ginseng extract reduce scopolamine-induced amnesia in a spatial learning task. J Psychiatr Neurosci 1999; 24: 442-52.

[14] Fulder S, Kataria M, Gethyn-Smith B. A double-blind clinical trial of Panax ginseng in aged subjects. Proceedings of the 4th International Ginseng Symposium, Daejeon, Korea. Seoul, Korea: Ginseng \& Tobacco Research Institute, 1984; pp. 215-23. 
[15] Neri M, Andermarcher E, Pradelli JM, Salvioli G. Influence of a double blind pharmacological trial on two domains of well-being in subjects with age associated memory impairment. Arch Gerontol Geriatr 1995; 21:241-52.

[16] Sørensen H, Sonne J. A double-masked study of the effects of ginseng on cognitive functions. Curr Ther Res Clin Exp 1996; 57: 959-68.

[17] Persson J, Bringlov E, Nilsson L, Nyberg L. The memoryenhancing effects of Ginseng and Ginkgo biloba in healthy volunteers. Psychopharmacology 2004; 172: 430-4.

[18] Lee ST, Chu K, Sim JY, Heo JH, Kim M. Panax Ginseng Enhances Cognitive Performance in Alzheimer Disease. Alzheimer Dis Assoc Disord 2008; 22(3): 222-6.

[19] Goel V, Lovlin R, Barton R, et al. Efficacy of a standardized Echinacea preparation (Echinilin) for the treatment of the common cold: a randomized, double-blind, placebo-controlled trial. J Clin Pharm Ther 2004; 29: 75-83.

[20] Wang M, Guilbert LJ, Li J, et al. A proprietary extract from North American ginseng (Panax quinquefolium) enhances IL-2 and IFNgamma productions in murine spleen cells induced by Con-A. Int Immunopharmacol 2004; 4: 311-5.

[21] Goel V, Lovlin R, Chang C, et al. A proprietary extract from the echinacea plant (Echinacea purpurea) enhances systemic immune response during a common cold. Phytother Res 2005; 19: 689-94.

[22] Huang, KC. The Pharmacology of Chinese Herbs. CRC Press, Louisville, Kentucky, USA, 1993; pp. 21-45.

[23] Bruneton, J. Pharmacognosy, Phytochemistry, Medicinal Plants, $2^{\text {nd }}$ ed. Intercept Ltd., UK Andover, 1993; pp. 563-5.

[24] Xu SL, Wu ZY. Development of the Clinical Memory Scale. Acta Psychol Sin 1986; 18: 100-8.

[25] Liu XJ, Zou YZ, Liu JQ, et al. Comparison between traditional and multimedia administration of the Clinical Memory Scale. Chin Ment Health J 2002; 16: 640-1.

[26] Xu WX, Guo YP. A clinical study of recovery of memories in 46 cases of cerebrovascular diseases. Zhonghua Nei Ke Za Zhi 1991; 30: $752-4$
[27] Li Y, Neng C, Ma J. Intelligence, memory, and event-related potentials in patients with multiple sclerosis. Zhonghua Nei Ke Za Zhi 1997; 36: 372-6.

[28] Zhang Y, Han B, Verhaeghen P, Nilsson L. Executive functioning in older adults with mild cognitive impairment: MCI has effects on planning, but not on inhibition. Aging Neuropsychol Cognit 2006; 1: $1-14$.

[29] Zhang ZX, Zahner GE, Roman GC, et al. Socio-demographic variation of dementia subtypes in China: Methodology and results of prevalence study in Beijing, Chengdu, Shanghai, and Xian. Neuroepidemiology 2006; 27 : 177-87.

[30] Xue S, Jia J. Genetic association between Ubiquitin Carboxyterminal Hydrolase-L1 gene S18Y polymorphism and sporadic Alzheimer's disease in a Chinese Han population. Brain Res 2006; 1087: 28-32.

[31] Liu F, Zhao X, Li J, Xu X. The influence of perceptual learning on drug abuser's cognition. Chin J Neurol Psychiatr Dis 2006; 32: 113-6.

[32] Scholey AB, Kennedy DO. Acute, dose-dependent cognitive effects of Ginkgo biloba, Panax ginseng and their combination in healthy young volunteers: differential interactions with cognitive demand. Hum Psychopharmacol Clin Exp 2002; 17: 35-44.

[33] Kennedy DO, Scholey AB, Wesnes KA. Modulation of cognition and mood following administration of single doses of Ginkgo biloba, ginseng, and a ginkgo/ginseng combination in healthy adults. Physiol Behav 2002; 75: 739-51.

[34] Kennedy DO, Haskell CF, Wesnes KA, Scholey AB. Improved cognitive performance in human volunteers following administration of guarana (Paullinia cupana) extract: comparison and interaction with Panax ginseng. Pharmacol Biochem Behav 2004; 79: 401-11.

[35] Reay JL, Kennedy DO, Scholey AB. Single doses of Panax ginseng (G115) reduce blood glucose levels and improve cognitive performance during sustained mental activity. J Psychopharmacol 2005; 19: 357-65. 